\title{
Pulse wave velocity as a measure of arterial stiffness in patients with familial hypercholesterolemia: a systematic review and meta-analysis
}

Željko Reiner ${ }^{1}$, Luis E. Simental-Mendía², Massimiliano Ruscica ${ }^{3}$, Niki Katsiki ${ }^{4}$, Maciej Banach5,6, Khalid Al Rasadi ${ }^{7}$, Tannaz Jamialahmadi ${ }^{8,9}$, Amirhossein Sahebkar ${ }^{10,11,12}$

${ }^{1}$ University Hospital Center Zagreb, Department of Internal Medicine, School of Medicine, University of Zagreb, Zagreb, Croatia

${ }^{2}$ Biomedical Research Unit, Mexican Social Security Institute, Durango, Mexico ${ }^{3}$ Department of Pharmacological and Biomolecular Sciences, Università degli Studi di Milano, Milan, Italy

${ }^{4}$ Second Propedeutic Department of Internal Medicine, Medical School, Aristotle University of Thessaloniki, Hippocration Hospital, Thessaloniki, Greece

${ }^{5}$ Department of Hypertension, WAM University Hospital in Lodz, Medical University of Lodz, Lodz, Poland

${ }^{6}$ Polish Mother's Memorial Hospital Research Institute (PMMHRI), Lodz, Poland

'Department of Clinical Biochemistry, Sultan Qaboos University Hospital, Muscat, Oman ${ }^{8} \mathrm{Halal}$ Research Center of IRI, FDA, Tehran, Iran

${ }^{9}$ Department of Nutrition, Faculty of Medicine, Mashhad University of Medical Sciences, Mashhad, Iran

${ }^{10}$ Neurogenic Inflammation Research Center, Mashhad University of Medical Sciences, Mashhad, Iran

${ }^{11}$ Biotechnology Research Center, Pharmaceutical Technology Institute, Mashhad University of Medical Sciences, Mashhad, Iran

${ }^{12}$ School of Pharmacy, Mashhad University of Medical Sciences, Mashhad, Iran

Submitted: 15 June 2019

Accepted: 22 June 2019

Arch Med Sci 2019; 15 (6): 1365-1374

DOI: https://doi.org/10.5114/aoms.2019.89450

Copyright $\odot 2019$ Termedia \& Banach

\section{Abstract}

Introduction: The aim of this meta-analysis was to establish whether vascular pulse wave velocity (PWV) as a measure of arterial stiffness is changed in patients with familial hypercholesterolemia $(\mathrm{FH})$.

Material and methods: Studies comparing PWV between patients with FH and controls were searched in PubMed-Medline, SCOPUS, Web of Science and Google Scholar databases (up to November 26, 2017). A meta-analysis was conducted using Comprehensive Meta-Analysis V2 software. A random-effects model (using the DerSimonian-Laird method) and the generic inverse variance method were used to compensate for the heterogeneity of studies concerning demographic characteristics and differences in the studies' design.

Results: This meta-analysis of 8 studies involving 317 patients with $\mathrm{FH}$ and 244 non-FH individuals did not suggest a significantly altered PWV in $\mathrm{FH}$ patients versus controls (weighted mean difference (WMD): $0.17 \mathrm{~m} / \mathrm{s}, 95 \%$ confidence interval $\left.(\mathrm{Cl}):-0.31,0.65, p=0.489 ; I^{2}=80.15 \%\right)$. The result was robust in the sensitivity analysis and its significance was not influenced after omitting each of the included studies from the meta-analysis. Subanalysis of 6 of these studies which had data on intima-media thickness (IMT) indicated an increased IMT in FH patients when compared with controls $\left(\mathrm{WMD}=0.03 \mathrm{~mm}, 95 \% \mathrm{Cl}: 0.003,0.06, p=0.034 ; I^{2}=48.95 \%\right)$. However, the effect size was sensitive to some of the included studies.

Conclusions: This meta-analysis suggests that $\mathrm{FH}$ patients do not have significantly altered PWV when compared with normocholesterolemic individuals. However, a subanalysis of studies in which IMT was measured indicated that IMT is increased in FH patients compared with controls.

\author{
Corresponding author: \\ Amirhossein Sahebkar \\ PharmD, PhD \\ Department of \\ Medical Biotechnology \\ School of Medicine \\ Mashhad University \\ of Medical Sciences \\ P.O. Box: $91779-48564$ \\ Mashhad, Iran \\ Phone: +985138002288 \\ Fax: +98 5138002287 \\ E-mail: \\ sahebkara@mums.ac.ir \\ amir_saheb2000@yahoo.com \\ amirhossein.sahebkar@uwa. \\ edu.au
}

Key words: familial hypercholesterolemia, arterial stiffness, pulse wave velocity, intima-media thickness. 


\section{Introduction}

Familial hypercholesterolemia (FH) is an autosomal co-dominant inherited disorder of lipoprotein metabolism characterized by markedly elevated plasma LDL cholesterol (LDL-C) concentration from birth [1]. Therefore, patients with $\mathrm{FH}$ have an increased risk of premature development of atherosclerosis, particularly atherosclerotic cardiovascular disease (ACVD) and/or coronary artery disease (CAD) [2]. Although heterozygous $\mathrm{FH}(\mathrm{HeFH})$ is one of the most common genetic disorders, and is associated with significant morbidity and mortality, it is very often underdiagnosed and massively undertreated [3-6]. Therefore, recently registries of $\mathrm{FH}$ are being developed to assess gaps in care and improve disease management and outcomes [7] and attempts are in progress to identify secondary treatment targets other than LDL-C - which is the most important and primary target - to reduce the risk of ACVD in these patients [8-11]. The prevalence of $\mathrm{FH}$ in the general population is estimated at around 1 in 200-500, while there are data indicating that in patients with established CAD, the prevalence of potential $\mathrm{FH}$ seems to be even $8.3 \% ; 7.5 \%$ in men and $11.1 \%$ in women [12]. $\mathrm{FH}$ is, in more than $90 \%$ of cases, caused by loss-offunction (LOF) mutations in the gene encoding $L D L$ receptor (LDLR), which have as a consequence a decreased cellular uptake of LDL particles and therefore significantly elevated plasma LDL-C concentrations [13]. Currently more than 1700 such mutations have been documented [14, 15]. However, mutations of other genes related to apolipoprotein B that affect the LDLR-binding domain of apolipoprotein $B$ as the most important apolipoprotein for LDL particles' uptake, and the gain-of-function (GOF) mutations of proprotein convertase subtilisin/kexin9 (PCSK9) - a serine protease essential for LDLR recycling - have also been identified and they result in an identical phenotype as patients with LDLR mutations $[2,16]$.

Atherosclerosis of the arteries causes loss of elasticity and increased rigidity, resulting in increased velocity of pulse waves since they travel faster in stiff arteries. Arterial stiffness is a robust predictor of all-cause and CVD mortality, fatal and non-fatal coronary events and fatal strokes [17-20]. As pulse wave velocity (PWV) is the most widely used and validated technique for estimating arterial stiffness, high PWV represents an early sign of arteriosclerosis/atherosclerosis [21].

Intima-media thickness (IMT), particularly carotid IMT, is recognized as a surrogate marker of atherosclerosis, given its predictive association with CAD [22]. Therefore, it was considered that the measurement of carotid IMT and/or screening for atherosclerotic plaques by carotid artery ultrasound can add information beyond assessment of traditional risk factors in asymptomatic adults at moderate CV risk [23]. However, the evidence that measurement of common carotid IMT can improve the risk prediction of CVD events is inconsistent [24]. Even the position paper from the European Society of Cardiology Working Group on peripheral circulation stated that it is still unclear whether a specific vascular biomarker (including IMT) is clearly superior and that a prospective study in which all vascular biomarkers are measured is still lacking [25].

The aim of this meta-analysis was to establish whether PWV as a marker of arterial stiffness is changed in patients with $\mathrm{FH}$ compared with non$\mathrm{FH}$ individuals. The secondary goal of this metaanalysis was to determine whether IMT is different in $\mathrm{FH}$ patients compared with non-FH subjects.

\section{Material and methods}

\section{Search strategy}

The study was designed according to the guidelines for systematic reviews and meta-analysis, the PRISMA statement [26]. SCOPUS, PubMed-Medline, Google Scholar and ISI Web of Science databases were searched using the following search terms in titles and abstracts: ("familial hypercholesterolemia" OR "familial hypercholesterolaemia" OR "familial hypercholesterolemic" OR "familial hypercholesterolaemic") AND ("pulse wave velocity" OR PWV OR aPWV OR cPWV OR fPWV OR CFPWV OR "arterial distensibility" OR "vascular distensibility" OR "aortic distensibility" OR "arterial stiffness" OR "arterial stiffening" OR "vascular stiffness" OR "vascular stiffening" OR "aortic stiffness" OR "aortic stiffening" OR "arterial compliance" OR "vascular compliance" OR "aortic compliance"). The wild-card term "*” was used to increase the sensitivity of the search strategy. No language restriction was used in the literature search. The literature was searched from inception to November 26, 2017.

\section{Study selection}

Original studies were included if they met the following inclusion criteria: (i) an observational study (i.e., a case-control, cross-sectional or cohort design), (ii) comparison of vascular PWV between patients with $\mathrm{FH}$ and controls without $\mathrm{FH}$, and (iii) presentation of information on PWV in each group. Studies with undefined control groups, duplicate or overlapped populations with a previous study, or reported PWV values in a single group were excluded.

\section{Data extraction}

The following data were basically needed: 1) first author's name, 2) year of publication, 
3) study location, 4) age and gender of patients,

5) number of patients (by gender if described), 6) study design, 7) type of $\mathrm{FH}$ (homozygous or heterozygous), 8) diagnostic criteria to define $\mathrm{FH}$, 9) IMT values, 10) type of vessel on which PWV was measured, and 11) PWV values.

\section{Quality assessment}

Methodological quality of the included studies was assessed using the Newcastle-Ottawa Scale (NOS) [27]. In this scale, three aspects of each eligible study are evaluated: (i) the selection of the studied patients (4 items), (ii) the comparability of the studied populations (one item) and (iii) the ascertainment of the exposure (3 items) in case-control studies or outcome of interest in cohort studies. A study can be awarded a maximum of one point for each item in the selection and exposure categories, whilst the comparability item can receive a maximum of two points.

\section{Quantitative data synthesis}

A meta-analysis was conducted using Comprehensive Meta-Analysis (CMA) V2 software [28]. All $P W V$ values were collated in $\mathrm{m} / \mathrm{s}$. Standard deviation (SD) of the mean difference was calculated using the following formula:

$$
S_{\text {pooled }}=\sqrt{\frac{\left(n_{1}-1\right) S_{1}^{2}+\left(n_{2}-1\right) S_{2}^{2}}{n_{1}+n_{2}-2}}
$$

Where $n_{1}$ and $n_{2}$ are population sizes of $\mathrm{FH}$ and control groups while $S 1$ and $S 2$ represent SD values of the respective groups.

If the outcome measures were reported in median and inter-quartile range, mean and SD values were estimated using the method as described by Wan et al. [29]. Where standard error of the mean (SEM) was only reported, SD was estimated using the following formula: SD $=$ SEM $\times$ sqrt $(n)$, where $\mathrm{n}$ is the number of subjects.

A random-effects model (using the DerSimonian-Laird method) and the generic inverse variance method were used to compensate for the heterogeneity of studies in terms of demographic characteristics of studied populations and also differences in the study design. Heterogeneity was quantitatively assessed using the $R$ index. Effect sizes were expressed as weighted mean difference (WMD) and $95 \%$ confidence interval $(\mathrm{Cl})$. In order to evaluate the influence of each study on the overall effect size, sensitivity analysis was conducted using the leave-one-out method, i.e. removing one study each time and repeating the analysis [30-32].

\section{Publication bias}

Potential publication bias was explored using visual inspection of Begg's funnel plot asymmetry,
Begg's rank correlation test and Egger's weighted regression test. The Duval \& Tweedie "trim and fill" method was used to adjust the analysis for the effects of publication bias [33].

\section{Results}

\section{Characteristics of the included studies}

Initially, 177 published studies were identified following a multiple database search. After screening of titles and abstracts and removing non-original studies $(n=69)$ and studies that did not meet the inclusion criteria $(n=91), 17$ full text articles were carefully assessed and reviewed for eligibility. Of these, 11 clinical trials were excluded for not measuring PWV $(n=9)$, because they were performed in a non-FH population $(n=1)$, or were uncontrolled studies $(n=1)$, thus leaving 8 eligible articles for the present meta-analysis (Figure 1).

A total of 317 patients with $\mathrm{FH}$ and 244 normocholesterolemic controls were included in this meta-analysis. Included studies were published between 2007 and 2016. FH populations from the following countries were included: Brazil, Greece, Italy, Russia, Poland, Taiwan and the UK. There were 4 studies performed on heterozygous and 4 on unspecified type of $\mathrm{FH}$ patients. Study design of selected studies was cross-sectional and case-control.

Concerning the diagnosis of $\mathrm{FH}$, four studies used gene mutation analysis, two studies used the Simon Broome criteria, one study used the Dutch Lipid Clinic Network criteria, and another study used the U.S.MEDPED criteria (Table I).

\section{PWV assay methods}

Different methods were used to measure the PWV. In this regard, one study used a VP-1000 device (Colin Corporation, Komaki, Japan) to measure the brachial-ankle PWV. One study determined the PWV with a Vicorder device (Skidmore medical, Bristol, UK) while another study used echo-tracking software (Aloka Prosound Alpha7, Hitachi-Aloka, Tokyo, Japan) and a $14 \mathrm{MHz}$ linear-type probe to assess the PWV. Two studies measured the PWV using the echo-tracking method with Aloka SSD-Alpha 10-Miro and the automatic system of ultrasound vascular evaluation. Furthermore, two other studies evaluated the PWV with a Complior device (Colson, Garges les Gonesses, France). Finally, Vlahos et al. [34] used echo-Doppler ultrasound (Ultrasound ATL, HDI 5000, Bothell, WA) to assess the PWV.

\section{Quality assessment of the included studies}

Most of the studies exhibited sufficient information concerning definition of cases and 


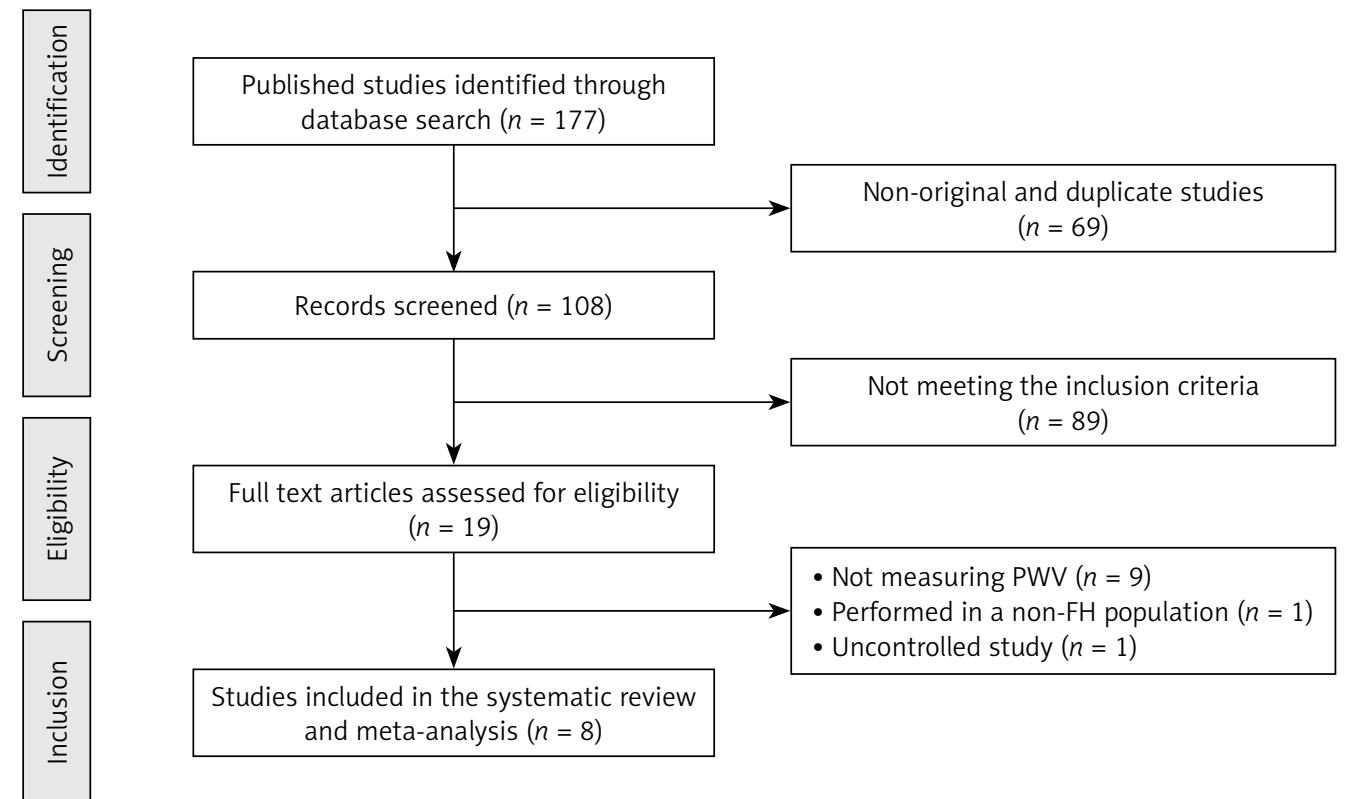

Figure 1. Flow chart of the number of studies identified and included in the meta-analysis

controls, but there was a lack of information regarding representativeness of the cases and selection of controls. Other parameters for quality assessment of the included studies are shown in Table II.

\section{Comparison of PWV between patients with $\mathrm{FH}$ and controls}

Overall, 8 studies compared PWV between patients with $\mathrm{FH}$ and normocholesterolemic controls. The meta-analysis did not suggest a significantly altered PWV in FH patients versus controls (WMD $=0.17 \mathrm{~m} / \mathrm{s}, 95 \% \mathrm{Cl}:-0.31,0.65, p=0.489 ; l^{2}=$ $80.15 \%$ ) (Figure 2). This result was robust in the sensitivity analysis and its significance was not influenced after omitting each of the included studies from the meta-analysis (Figure 2). In the subgroup analysis, there was no significant difference between subgroups of studies in patients $\leq 20$ years $(\mathrm{WMD}=0.41 \mathrm{~m} / \mathrm{s}, 95 \% \mathrm{Cl}:-0.95,1.76$, $\left.p=0.557 ; l^{2}=94.41 \%\right)$ and $>20$ years (WMD $=0.07 \mathrm{~m} / \mathrm{s}, 95 \% \mathrm{Cl}:-0.39,0.53, p=0.758 ; l^{2}=$ $62.72 \%$ ) (between-group $p=0.647$ ). Likewise, the estimated effect size was not significantly different between patients with $\mathrm{FH}$ and controls in the subgroup of studies in confirmed HeFH patients $(\mathrm{WMD}=0.40 \mathrm{~m} / \mathrm{s}, 95 \% \mathrm{Cl}:-0.29,1.09, p=0.253$; $\left.r^{2}=83.78 \%\right)$.

Among the included studies, 6 studies also assessed IMT. Subanalysis of these studies indicated an increased IMT in FH patients when compared with controls (WMD $=0.03 \mathrm{~mm}, 95 \% \mathrm{Cl}: 0.003$, $0.06, p=0.034 ; l^{2}=48.95 \%$ ) (Figure 3). However, the effect size was sensitive to some of the included studies, as shown in Figure 3.

\section{Publication bias}

There was no evidence of publication bias according to the results of Egger's linear regression (intercept $=-1.32$, standard error $=2.16,95 \% \mathrm{Cl}$ : -6.61, 3.97, $t=0.61, \mathrm{~d} f=6$, two-tailed $p=0.565$ ) and Begg's rank correlation tests (Kendall's $\tau$ with continuity correction $=-0.04, z=0.12$, two-tailed $p=0.902$; Figure 4). The funnel plot of the study standard error by effect size (WMD) was slightly asymmetric. This asymmetry was addressed by imputing one potentially missing study using the "trim and fill" method. After imputation, the effect size was changed to $0.23(95 \% \mathrm{Cl}:-0.25,0.71)$ and remained non-significant.

\section{Discussion}

To our knowledge this is the first meta-analysis evaluating PWV as a measure of arterial stiffness in patients with $\mathrm{FH}$. The results of this meta-analysis suggest that $\mathrm{FH}$ patients do not have significantly altered PWV compared with controls. However, a subanalysis of studies in which IMT was measured indicates that IMT is increased in $\mathrm{FH}$ patients when compared with controls.

This meta-analysis did not include the most recently published results of a study performed on 245 patients with $\mathrm{FH}$, which suggested a different conclusion, i.e. that arterial stiffness assessed by the PWV was significantly associated with the presence of coronary heart disease in patients with FH [35]. Nevertheless, there was no comparison with normocholesterolemic controls in the study published by Tada et al. [35]. Another recent study on 66 patients with $\mathrm{FH}$ and their 57 first-degree relatives without $\mathrm{FH}$ demonstrated that treat- 


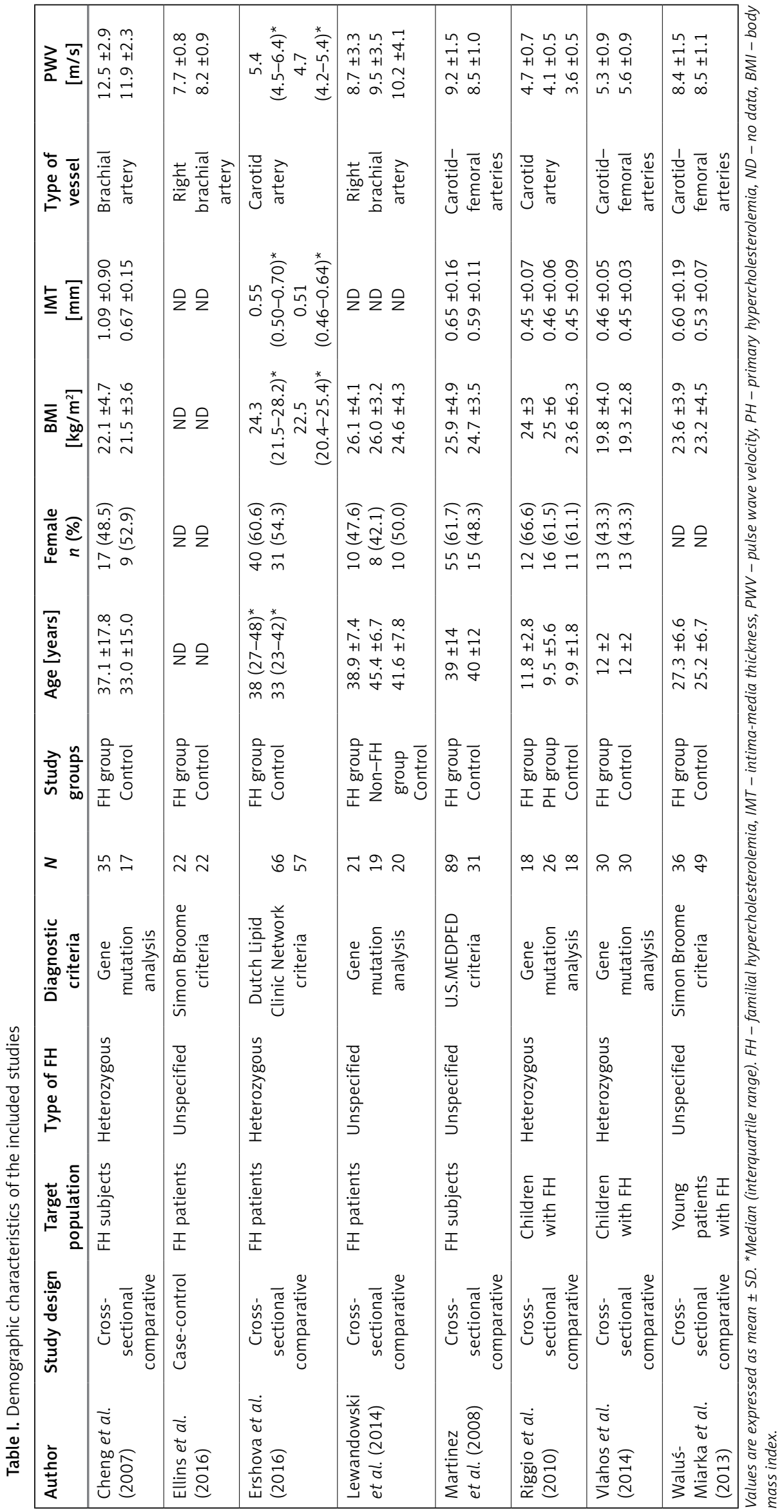




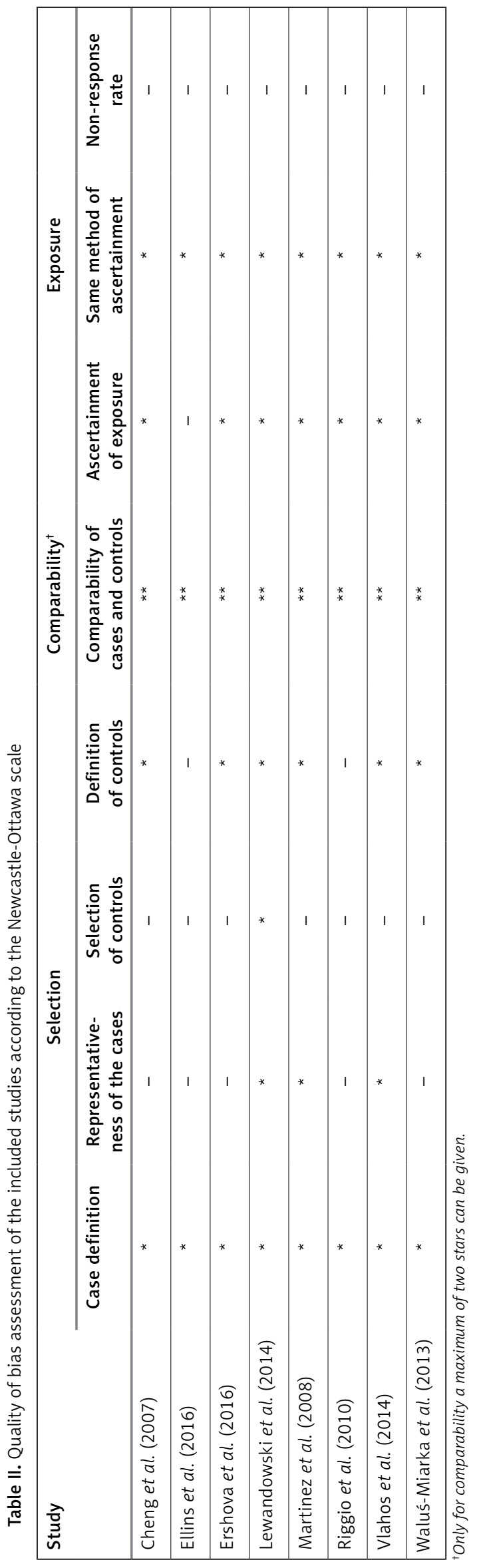

ment-naïve FH patients had stiffer carotid arteries than their relatives but showed no difference in aortic stiffness [36]. Furthermore, in another relatively small study (81 FH patients compared with normal subjects), these markers poorly correlated among each other in univariate analysis and this correlation disappeared after adjustment for confounders [37]. Other authors could also not find any significant difference in arterial stiffness (assessed by pulse-wave analysis using the echo-tracking method and photoplethysmographic pulse waveform analysis) between patients with and without $\mathrm{FH}$ [38].

There are reports that arterial stiffness measured by PWV, but not IMT, is increased in untreated hypercholesterolemic children (almost half of them had $\mathrm{FH}$ ) when compared with age- and sexmatched controls [39]. When mentioning studies in children with $\mathrm{FH}$, a relatively recent study found no significant difference either in arterial stiffness or in carotid IMT between HeFH children and their sex- and age-matched controls without FH [34]. However, a very recent study showed that in $\mathrm{HeFH}$ children carotid IMT was significantly greater at baseline when compared with unaffected siblings [40]. The same study indicated that treatment with rosuvastatin for two years resulted in significantly less progression of increased carotid IMT in children with $\mathrm{HeFH}$ than untreated unaffected siblings and, as a result, no difference in carotid IMT could be detected between these two groups after 2 years. This confirmed earlier studies indicating that the difference in carotid IMT between children with $\mathrm{FH}$ and their unaffected siblings may be significant as early as at 8 years of age, that long-term statin treatment (lasting 10 years) initiated during childhood in patients with $\mathrm{FH}$ was associated with normalization of carotid IMT progression, and that earlier statin initiation was associated with thinner carotid IMT at follow-up [41, 42].

It could be supposed that long-lasting lipid-lowering treatment might improve PWV in adult patients with FH. Indeed, it was shown that 1 year of cholesterol lowering therapy with statins (simvastatin, atorvastatin $40-80 \mathrm{mg}$ day) in FH patients can decrease the wall stiffness in the carotid and femoral arterial wall and thickness in the common carotid artery [43].

It is well known that adult patients with $\mathrm{FH}$ are not at moderate but either at high or at very high ACVD risk, so there is no point in screening them by carotid IMT measurement in order to improve their risk assessment [44-46]. Therefore, the finding of this meta-analysis, that IMT is increased in FH patients compared with controls, is absolutely logical and fits well with the common knowledge about $\mathrm{FH}$. Moreover, a recent study proved that $\mathrm{FH}$ patients with a monogenic cause of the disease 


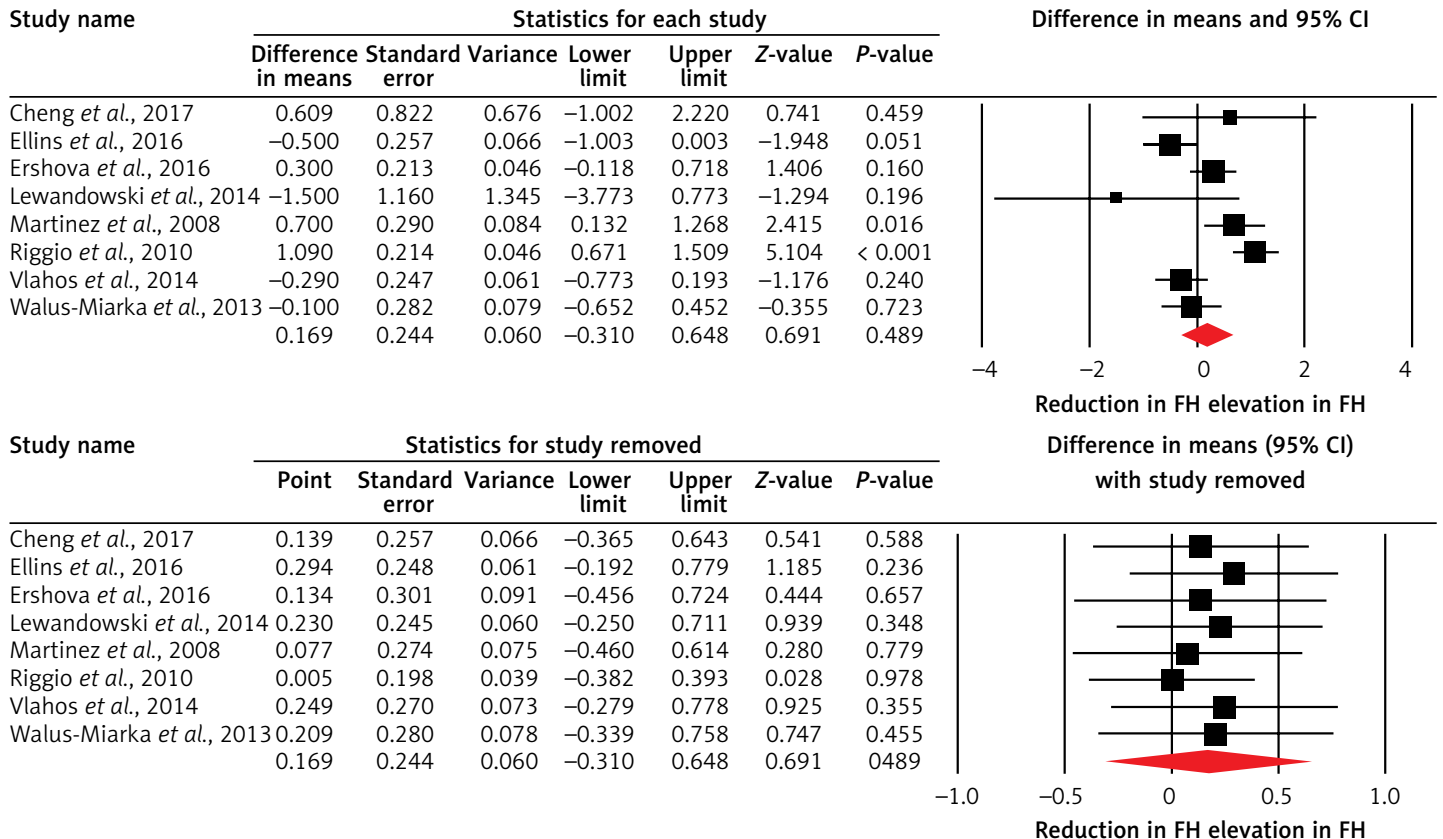

Figure 2. Forest plot displaying weighted mean difference and $95 \%$ confidence intervals of pulse wave velocity between patients with familial hypercholesterolemia and unaffected controls. Lower plot shows leave-one-out sensitivity analysis

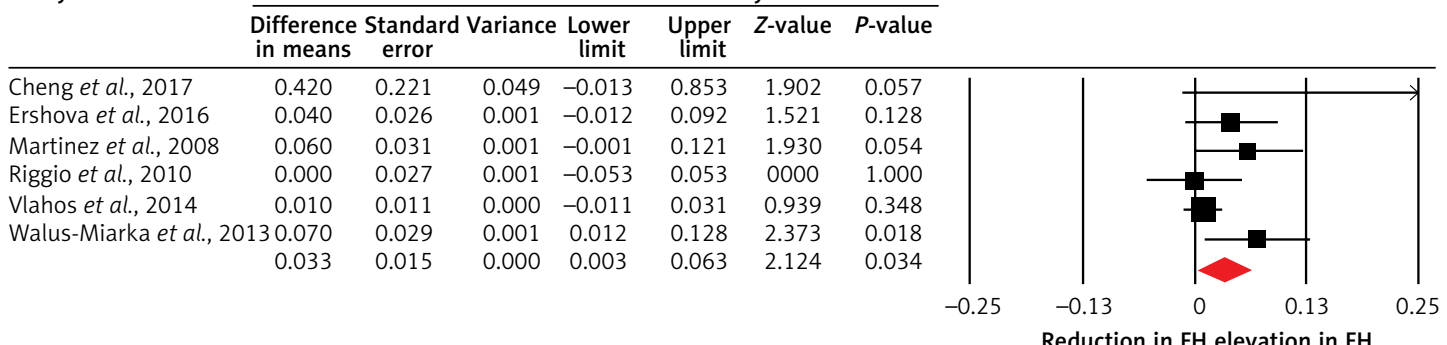

Statistics for study removed

Reduction in $\mathrm{FH}$ elevation in $\mathrm{FH}$

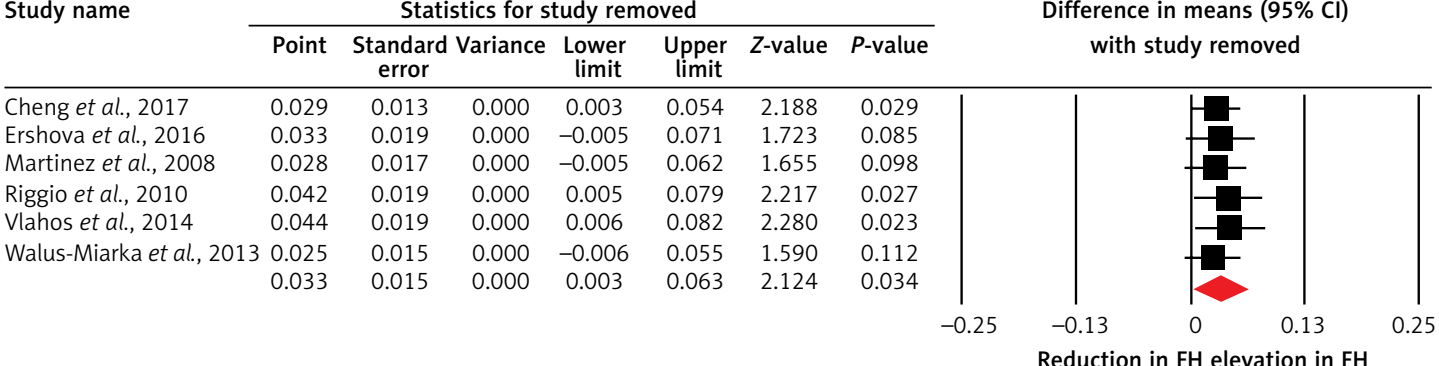

Figure 3. Forest plot displaying weighted mean difference and $95 \%$ confidence intervals of intima-media thickness between patients with familial hypercholesterolemia and unaffected controls. Lower plot shows leave-one-out sensitivity analysis

have a greater carotid IMT and more severe coronary preclinical atherosclerosis than those with a polygenic etiology [47].

Only one meta-analysis which investigated IMT in $\mathrm{FH}$ patients compared to normolipidemic controls has been published, 7 years ago. In this metaanalysis, in $\mathrm{FH}$ patients both carotid and femoral IMT values were higher [48]. Nevertheless, this meta-analysis was aimed more at proving that treatment with statins can improve arterial function and structure in $\mathrm{FH}$ patients in a treatment intensity-related manner. In this context it is interesting to mention that one study proved that not only carotid IMT but also carotid plaques did not differ between long-term statin-treated HeFH patients and healthy controls, suggesting that long-term treatment in these patients can reduce carotid atherosclerosis to the degree of a healthy 


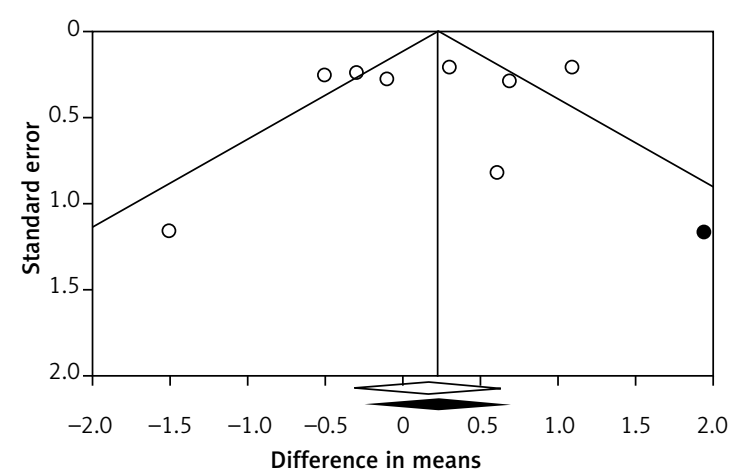

Figure 4. Funnel plot detailing publication bias. Open diamond represents observed effect size; closed diamond represents imputed effect size
Aventis and Lilly. NK has given talks, attended conferences and participated in trials sponsored by Amgen, Angelini, Astra Zeneca, Boehringer Ingelheim, Galenica, MSD, Novartis, Novo Nordisk, Sanofi and WinMedica. KR received a research grant from Sanofi, and served on the speaker's bureau and as an advisory board member for Sanofi, Astra Zeneca and Pfizer. ZR has received honoraria from Sanofi Aventis and Akcea. The authors have no other relevant affiliations or financial involvement with any organization or entity with a financial interest in or financial conflict with the subject matter or materials discussed in the manuscript apart from those disclosed.

population [49]. These findings strongly suggest that measuring carotid IMT during follow-up of statin-treated $\mathrm{FH}$ patients has limited value [49]. When mentioning measurement of carotid plaques, a very recent study on 225 patients with FH showed that carotid plaque score determined by carotid ultrasonography may provide superior risk stratification in patients with $\mathrm{FH}$ compared with carotid IMT [50].

Our meta-analysis has several limitations. Different scores for $\mathrm{FH}$ diagnosis as well as different methods for PWV estimation were used in different studies included in the present meta-analysis and there was a lack of information about the duration of lipid-lowering therapy and type of treatment (i.e. statin type and dose, statin and ezetimibe combined treatment, apheresis, PCSK9 inhibitors). These data are important as statins may improve PWV and arterial stiffness [51,52] while the information on novel lipid-lowering therapies including PCSK9 inhibitors is scant. In this context, PCSK9 inhibitors have been suggested to have additional effects $[53,54]$ beyond their well-known lipid-lowering properties $[55,56]$. Similarly, antihypertensive drugs may differentially affect PWV based on the type and duration of treatment [57-60].

In conclusion, the present meta-analysis suggests that FH patients do not have significantly altered PWV as a measure of arterial stiffness when compared with normocholesterolemic controls. However, a subanalysis of studies, in which IMT was measured, indicates that IMT is increased in FH patients when compared with controls. Given the prevalence and burden of FH [61-63], additional studies are suggested to assess IMT as a risk predictor in FH individuals. Obviously, larger studies evaluating $\mathrm{PWV}$ in $\mathrm{FH}$ patients compared with controls in order to elucidate the impact of $\mathrm{FH}$ on arterial stiffness as measured by PWV, if any, are needed.

\section{Conflict of interest}

$M B$ has served on the speaker's bureau and as an advisory board member for Amgen, Sanofi,

\section{References}

1. Catapano AL, Graham I, De Backer G, et al. 2016 ESC/ EAS guidelines for the management of dyslipidaemias. Eur Heart I 2016; 37: 2999-3058.

2. Reiner Ž. Management of patients with familial hypercholesterolaemia. Nat Rev Cardiol 2015; 12: 565-75.

3. Pećin I, Hartgers ML, Hovingh GK, et al. Prevention of cardiovascular disease in patients with familial hypercholesterolaemia: the role of PCSK9 inhibitors. Eur J Prev Cardiol 2017; 24: 1383-401.

4. Vallejo-Vaz AJ, Kondapally Seshasai SR, Cole D, et al. Familial hypercholesterolaemia: a global call to arms. Atherosclerosis 2015; 243: 257-59.

5. Rynkiewicz A, Cybulska B, Banach M, et al. Management of familial heterozygous hypercholesterolemia: position paper of the Polish Lipid Expert Forum. J Clin Lipidol 2013; 7: 217-21.

6. Myśliwiec M, Walczak M, Małecka-Tendera E, et al. Management of familial hypercholesterolemia in children and adolescents. Position paper of the Polish Lipid Expert Forum. J Clin Lipidol 2014; 8: 173-80.

7. EAS Familial Hypercholesterolaemia Studies Collaboration, Vallejo-Vaz AJ, Akram A, et al. Pooling and expanding registries of familial hypercholesterolaemia to assess gaps in care and improve disease management and outcomes: rationale and design of the global EAS Familial Hypercholesterolaemia Studies Collaboration. Atheroscler Suppl 2016; 22: 1-32.

8. Ganjali S, Momtazi AA, Banach M, et al. HDL abnormalities in familial hypercholesterolemia: focus on biological functions. Prog Lipid Res 2017; 67: 16-26.

9. Ganjali S, Momtazi-Borojeni AA, Banach M, et al. HDL functionality in familial hypercholesterolemia: effects of treatment modalities and pharmacological interventions. Drug Discov Today 2018; 23: 171-80.

10. Mollazadeh H, Carbone F, Montecucco F, et al. Oxidative burden in familial hypercholesterolemia. J Cell Physiol 2018; 233: 5716-25.

11. Momtazi AA, Banach M, Pirro M, et al. MicroRNAs: new therapeutic targets for familial hypercholesterolemia? Clin Rev Allergy Immunol 2018; 54: 224-33.

12. De Backer G, Besseling J, Chapman JG, et al. Prevalence and management of familial hypercholesterolaemia in coronary patients: an analysis of EUROASPIRE IV, a study of the European Society of Cardiology. Atherosclerosis 2015; 241: 169-75.

13. Goldstein JL, Brown MS. Familial hypercholesterolemia: identification of a defect in the regulation of 3-hydroxy-3-methylglutaryl coenzyme A reductase activity 
associated with overproduction of cholesterol. Proc Natl Acad Sci USA 1973; 70: 2804-8.

14. www.ucl.ac.uk/Idlr/LOVDv.1.1.0/

15. Fairoozy RH, Futema M, Vakili R, et al. The genetic spectrum of familial hypercholesterolemia $(\mathrm{FH})$ in the Iranian population. Sci Rep 2017; 7: 17087.

16. Abifadel M, Varret M, Rabès JP, et al. Mutations in PCSK9 cause autosomal dominant hypercholesterolemia. Nat Genet 2003; 34: 154-56.

17. Cecelja M, Chowienczyk P. Role of arterial stiffness in cardiovascular disease. JRSM Cardiovasc Dis 2012; 1: pii: cvd.2012.012016.

18. Vlachopoulos C, Aznaouridis K, Stefanadis C. Prediction of cardiovascular events and all-cause mortality with arterial stiffness: a systematic review and meta-analysis. J Am Coll Cardiol 2010; 55: 1318-27.

19. Yamashina A, Tomiyama $\mathrm{H}$, Takeda $\mathrm{K}$, et al. Validity, reproducibility, and clinical significance of noninvasive brachial-ankle pulse wave velocity measurement. Hypertens Res 2002; 25: 359-64.

20. Zieman SJ, Melenovsky V, Kass DA. Mechanisms, pathophysiology, and therapy of arterial stiffness. Arterioscler Thromb Vasc Biol 2005; 25: 932-43.

21. Laurent S, Cockcroft J, Van Bortel L, et al. Expert consensus document on arterial stiffness: methodological issues and clinical applications. Eur Heart J 2006; 27: 2588-605.

22. Stein JH, Korcarz CE, Hurst RT, et al. Use of carotid ultrasound to identify subclinical vascular disease and evaluate cardiovascular disease risk: a consensus statement from the American Society of Echocardiography Carotid Intima-Media Thickness Task Force. Endorsed by the Society for Vascular Medicine. J Am Soc Echocardiogr 2008; 21: 93-111.

23. Perk J, De Backer G, Gohlke H, et al. European Guidelines on cardiovascular disease prevention in clinical practice (version 2012). The Fifth Joint Task Force of the European Society of Cardiology and Other Societies on Cardiovascular Disease Prevention in Clinical Practice (constituted by representatives of nine societies and by invited experts). Eur Heart J 2012; 33: 1635-701.

24. Den Ruijter HM, Peters SA, Anderson TJ, et al. Common carotid intima-media thickness measurements in cardiovascular risk prediction: a meta-analysis. JAMA 2012; 308: 796-803.

25. Vlachopoulos C, Xaplanteris P, Aboyans V, et al. The role of vascular biomarkers for primary and secondary prevention. A position paper from the European Society of Cardiology Working Group on peripheral circulation: Endorsed by the Association for Research into Arterial Structure and Physiology (ARTERY) Society. Atherosclerosis $2015 ; 241$ : 507-32.

26. Moher D, Liberati A, Tetzlaff J, et al. Preferred reporting items for systematic reviews and meta-analyses: the PRISMA statement. BMJ 2009; 339: b2535.

27. Wells GA, Shea B, O'Connell D, et al. The Newcastle-Ottawa Scale (NOS) for assessing the quality of nonrandomized studies in meta-analysis. 2011.

28. Borenstein M, Hedges L, Higgins J, et al. Comprehensive Meta-analysis Version 2. Biostat 2005, Englewood, NJ.

29. Wan X, Wang W, Liu J, et al. Estimating the sample mean and standard deviation from the sample size, median, range and/or interquartile range. BMC Med Res Methodol 2014; 14: 135.

30. Sahebkar A, Kotani K, Serban C, et al. Statin therapy reduces plasma endothelin-1 concentrations: a meta- analysis of 15 randomized controlled trials. Atherosclerosis 2015; 241: 433-42.

31. Sahebkar A. Does PPARgamma2 gene Pro12Ala polymorphism affect nonalcoholic fatty liver disease risk? Evidence from a meta-analysis. DNA Cell Biol 2013; 32: 188-98.

32. Sahebkar A, Serban C, Mikhailidis DP, et al.; Lipid and Blood Pressure Meta-analysis Collaboration (LBPMC) Group. Association between statin use and plasma D-dimer levels. A systematic review and meta-analysis of randomised controlled trials. Thromb Haemost 2015; 114: 546-57.

33. Duval S, Tweedie R. Trim and fill: a simple funnel-plotbased method of testing and adjusting for publication bias in meta-analysis. Biometrics 2000; 56: 455-63.

34. Vlahos AP, Naka KK, Bechlioulis A, et al. Endothelial dysfunction, but not structural atherosclerosis, is evident early in children with heterozygous familial hypercholesterolemia. Pediatr Cardiol 2014; 35: 63-70.

35. Tada H, Kawashiri MA, Nohara A, et al. Assessment of arterial stiffness in patients with familial hypercholesterolemia. J Clin Lipidol 2018; 12: 397-402.e2.

36. Ershova Al, Meshkov AN, Rozhkova TA, et al. Carotid and aortic stiffness in patients with heterozygous familial hypercholesterolemia. PLoS One 2016; 11: e0158964.

37. Martinez LR, Miname MH, Bortolotto LA, et al. No correlation and low agreement of imaging and inflammatory atherosclerosis' markers in familial hypercholesterolemia. Atherosclerosis 2008; 200: 83-8.

38. Lewandowski P, Romanowska-Kocejko M, Węgrzyn A, et al. Noninvasive assessment of endothelial function and vascular parameters in patients with familial and nonfamilial hypercholesterolemia. Pol Arch Med Wewn 2014; 124: 516-24.

39. Riggio S, Mandraffino G, Sardo MA, et al. Pulse wave velocity and augmentation index, but not intima-media thickness, are early indicators of vascular damage in hypercholesterolemic children. Eur J Clin Invest 2010; 40: 250-7.

40. Braamskamp MJAM, Langslet G, McCrindle BW, et al. Effect of rosuvastatin on carotid intima-media thickness in children with heterozygous familial hypercholesterolemia: The CHARON Study (Hypercholesterolemia in Children and Adolescents Taking Rosuvastatin Open Label). Circulation 2017; 136: 359-66.

41. Kusters DM, Avis HJ, de Groot E, et al. Ten-year follow-up after initiation of statin therapy in children with familial hypercholesterolemia. JAMA 2014; 312: 1055-7.

42. Kusters DM, Wiegman A, Kastelein JJ, et al. Carotid intima-media thickness in children with familial hypercholesterolemia. Circ Res 2014; 114: 307-10.

43. Smilde TJ, van den Berkmortel FW, Wollersheim $\mathrm{H}$, et al. The effect of cholesterol lowering on carotid and femoral artery wall stiffness and thickness in patients with familial hypercholesterolaemia. Eur J Clin Invest 2000; 30: 473-80.

44. Reiner Ž. Impact of early evidence of atherosclerotic changes on early treatment in children with familial hypercholesterolemia. Circ Res 2014; 114: 233-5.

45. Reiner Ž. Statins in the primary prevention of cardiovascular disease. Nat Rev Cardiol 2013; 10: 453-64.

46. Reiner Ž. Statins for primary prevention: problems with cardiovascular-risk estimation? Nat Rev Cardiol 2013; 10: 547.

47. Sharifi M, Higginson E, Bos S, et al. Greater preclinical atherosclerosis in treated monogenic familial hypercho- 
lesterolemia vs. polygenic hypercholesterolemia. Atherosclerosis 2017; 263: 405-11.

48. Masoura C, Pitsavos C, Aznaouridis K, et al. Arterial endothelial function and wall thickness in familial hypercholesterolemia and familial combined hyperlipidemia and the effect of statins. A systematic review and metaanalysis. Atherosclerosis 2011; 214: 129-38.

49. Bos S, Duvekot MHC, ten Kate GJR, et al. Carotid artery plaques and intima medial thickness in familial hypercholesteraemic patients on long-term statin therapy: a case control study. Atherosclerosis 2017; 256: 62-6.

50. Tada H, Kawashiri MA, Okada H, et al. Assessments of carotid artery plaque burden in patients with familial hypercholesterolemia. Am J Cardiol 2017; 120: 1955-60.

51. Upala S, Wirunsawanya K, Jaruvongvanich V, et al. Effects of statin therapy on arterial stiffness: a systematic review and meta-analysis of randomized controlled trial. Int I Cardiol 2017; 227: 338-41.

52. Sahebkar A, Pećin I, Tedeschi-Reiner E, et al. Effects of statin therapy on augmentation index as a measure of arterial stiffness: a systematic review and meta-analysis. Int J Cardiol 2016; 212: 160-8.

53. Momtazi-Borojeni AA, Nik ME, Jaafari MR, Banach $M$, Sahebkar A. Effects of immunization against PCSK9 in an experimental model of breast cancer. Arch Med Sci 2019; 15: 570-9.

54. Momtazi-Borojeni AA, Nik ME, Jaafari MR, Banach $M$, Sahebkar A. Potential anti-tumor effect of a nanoliposomal antiPCSK9 vaccine in mice bearing colorectal cancer. Arch Med Sci 2019; 15: 559-69.

55. Banach M, Jankowski P, Jóźwiak J, et al. PoLA/CFPiP/PCS guidelines for the management of dyslipidaemias for family physicians 2016. Arch Med Sci 2017; 13: 1-45.

56. Banach M, Penson PE. What have we learned about lipids and cardiovascular risk from PCSK9 inhibitor outcome trials: ODYSSEY and FOURIER? Cardiovasc Res 2019; 115: e26-31.

57. Koumaras C, Tziomalos K, Stavrinou E, et al. Effects of renin-angiotensin-aldosterone system inhibitors and beta-blockers on markers of arterial stiffness. J Am Soc Hypertens 2014; 8: 74-82.

58. Koumaras C, Tzimou M, Stavrinou E, et al. Role of antihypertensive drugs in arterial 'de-stiffening' and central pulsatile hemodynamics. Am J Cardiovasc Drugs 2012; 12: 143-56.

59. Shahin Y, Khan JA, Chetter I. Angiotensin converting enzyme inhibitors effect on arterial stiffness and wave reflections: a meta-analysis and meta-regression of randomised controlled trials. Atherosclerosis 2012; 221: 18-33.

60. Niu W, Qi Y. A meta-analysis of randomized controlled trials assessing the impact of beta-blockers on arterial stiffness, peripheral blood pressure and heart rate. Int J Cardiol 2016; 218: 109-17.

61. EAS Familial Hypercholesterolaemia Studies Collaboration, Vallejo-Vaz AJ, De Marco M, Stevens CAT, et al. Overview of the current status of familial hypercholesterolaemia care in over 60 countries - The EAS Familial Hypercholesterolaemia Studies Collaboration (FHSC). Atherosclerosis 2018; 277: 234-55.

62. Dyrbuś K, Gąsior M, Desperak P, Osadnik T, Nowak J, Banach $M$. The prevalence and management of familial hypercholesterolemia in patients with acute coronary syndrome in the polish tertiary centre: results from the TERCET registry with 19,781 individuals. Atherosclerosis 2019; 288: 33-41.
63. Banach M, Mazidi M, Mikhailidis DP, et alF. Association between phenotypic familial hypercholesterolaemia and telomere length in US adults: results from a multi-ethnic survey. Eur Heart J 2018; 39: 3635-40. 